\title{
Propiedades psicométricas de la versión en español del Acbievement Goals Questionnaire
}

Francisco Ruiz-Juan*

Facultad de Ciencias del Deporte, Universidad de Murcia

\begin{abstract}
Resumen: El objetivo de este trabajo es la adaptación y comprobación de las propiedades psicométricas Achievement Goals Questionnaire ( $A G Q$ ) en español. Para ello se realizan dos estudios independientes. El objetivo del primero es presentar datos psicométricos preliminares (muestra piloto: 247 estudiantes). Expuesto el proceso de traducción, adaptación y validez de contenido de los ítems, se efectúa un análisis estadístico de éstos, la exploración de la estructura dimensional y análisis de la fiabilidad del instrumento. En el segundo (muestra: 2168 estudiantes), el objetivo es analizar con procedimientos confirmatorios la estructura interna del instrumento en tres países de habla hispana: España, México y Costa Rica. La versión en español del $A G Q$ mostró niveles adecuados de consistencia interna, estabilidad temporal, correlación entre la puntuación de los ítems y la puntuación total en cada uno de los componentes. Igualmente, se aportan evidencias de su validez de constructo. Se obtuvieron diferencias de sexo. Estos hallazgos apoyan el uso de la versión en español del $A G Q$ para evaluar las orientaciones de meta de logro que tienen los alumnos en las clases de Educación Física.
\end{abstract}

Palabras clave: Orientaciones de meta; propiedades psicométricas; metas de logro; estudio instrumental.
Title: Psychometric properties of the Spanish version of the Achievement Goals Questionnaire.

Abstract: In this instrumental paper, the aim was the adaptation and testing of the psychometric properties of the Achievement Goals Questionnaire $(A G Q)$ in Spanish. To this end, we conducted two independent studies. The aim of the first was to present the first preliminary psychometric data (pilot sample of 247 students). Explained the process of translation, adaptation and content validity of items, we carried out a statistical analysis of these, an exploration of dimensional structure and analysis of the reliability of the instrument. In the second study (sample of 2168 students), the aim was to analyze the internal structure of the instrument with confirmatory procedures in three Spanish-speaking countries: Spain, Mexico, and Costa Rica. The Spanish version of the $A G Q$ showed acceptable levels of internal consistency, temporal stability, correlation score between the items and the total score in each of the components. We also provides evidences of construct validity. Sex differences were obtained. These findings support the use of the Spanish version of the AGQ to assess achievement goal orientations with students in physical education classes.

Key words: Goal orientations; psychometric properties; achievement goals; instrumental study.

\section{Introducción}

En Educación Física, como en otros contextos, es necesario conocer las razones por las cuales el alumnado se implica y está motivado en las clases de Educación Física, es decir, qué espera conseguir al realizar una determinada actividad, cuál es el valor de esa actividad y cómo define el éxito y el fracaso en una situación de clase (Peiró y Sanchís, 2004).

Desde hace algo más de dos décadas, los teóricos de las metas de logro han adoptado un enfoque cognitivo social para estudiar la motivación y el comportamiento de los individuos (Ames, 1992, Dweck, 1986; Elliot, 1997; Nicholls, 1989), que considera que las metas de un individuo se basan en demostrar competencia y habilidad en diferentes contextos de logro (Dweck, 1986; Maehr y Braskamp, 1986; Ries, 2011), como el educativo y/o deportivo. Según este enfoque, los sujetos definen el éxito o fracaso en función de sus metas. Ha quedado demostrado que la teoría de metas de logro puede explicar y predecir creencias, respuestas y comportamiento en situaciones de logro (Wang, Biddle y Elliot, 2007). Igualmente, está ampliamente reconocido que hay factores personales y situacionales que influyen en la adopción de metas, si bien la gran mayoría de estos trabajos se centran en disposiciones personales y no tanto en aspectos contextuales de metas (Wang, Chia, Chatzisarantis y Lim, 2010). Así, los sujetos adquieren, por influencia social, dos orientaciones o formas de concebir el éxito: la orientación a la tarea o a la maestría, en la que el éxito viene definido co-

* Dirección para correspondencia [Correspondence address]: Francisco Ruiz-Juan. Facultad de Ciencias del Deporte. C/ Argentina s/n. 30720 Santiago de la Ribera, Murcia (España). E-mail: fruizj@,um.es mo el dominio de la tarea y el progreso personal, y la orientación al ego o al rendimiento, en la que el éxito se define como superación a los rivales y demostración de mayor capacidad (Nicholls, 1984).

Cuando los deportistas presentan altos valores en la orientación a la tarea, comúnmente se centran en adquirir maestría, por lo tanto, la percepción de la habilidad es autoreferenciada y el énfasis se pone en el esfuerzo, en el desarrollo y la mejora de las habilidades. En el contexto de la educación física, el alumnado está muy motivado, sin necesidad de recompensas externas o amenazas, porque la mejor recompensa es el logro en sí mismo (Ruiz-Juan, Piéron y Zamarripa, 2011).

En la orientación hacia el ego, el concepto de habilidad es normativo. Es decir, se toma a los demás como punto de referencia, el éxito significa hacer las cosas mejor que el resto de los compañeros y lo demuestran o bien ganando, puntuando más que el resto de compañeros en cualquier actividad, o realizando una ejecución que se considera buena con base a unos patrones normativos. Los deportistas con altos valores en la orientación al ego, centran su interés en demostrar que son mejores que los demás, les preocupa la habilidad normativa y destacar sobre otros, o hacer notar que ellos son iguales a los otros pero superan las tareas con menor esfuerzo. En este último caso se utilizan estándares de comparación social para hacer juicios de habilidad y resultado (Balaguer, Castillo, Duda y García-Merita, 2011).

El alumnado orientado al ego corre el riesgo de mostrar conductas de inadaptación como, por ejemplo, la resistencia a esforzarse ante actividades que requieren una alta exigencia y la tendencia a evitar actividades que le suponen un reto. Como su criterio de éxito depende de la acción de los demás, la mejora o el progreso en una tarea no es suficiente 
para producirle sentimientos de competencia (Ruiz-Juan y Piéron, 2013). En este sentido, aquellos jóvenes orientados al ego cuya percepción de capacidad ante una actividad propuesta sea baja son los más vulnerables, ya que presagian el fracaso, la vergüenza y la ansiedad, así como otras emociones negativas. Con el fin de evitar sufrir estas emociones, intentan por todos los medios no realizar la actividad propuesta, o se esfuerzan lo mínimo o bien dejan de asistir a las clases (Papaioannou, Marsh y Theodorakis, 2004). De ahí que los patrones de inadaptación en contextos de logro, como son el abandono de una práctica física y el deterioro de la ejecución, serán consecuencias conductuales de una orientación al ego cuando los jóvenes no confían en su capacidad o competencia (Peiró y Sanchís, 2004).

Gran parte de las investigaciones que han abordado el estudio de las metas de logro (Ames y Archer, 1988; Duda y Whitehead, 1998; Goudas y Biddle, 1994; Mitchell, 1996; Newton, Duda y Yin, 2000; Ntoumanis y Biddle, 1999; Papaioannou, 1994) no han considerado los avances recientes de esta teoría que incorporan las metas de evitación (Elliot y Church, 1997). La distinción de aproximación-evitación tiene una rica historia en la literatura de la motivación de logro, pero las investigaciones anteriores de meta de logro se centraron sólo en las formas de aproximación (Elliot, 2005). Dweck (1986) muestra la existencia de dos metas de logro que eran bipolares, mientras que Nicholls (1989) establece que estas dos dimensiones eran independientes y que se encontraban relacionadas ortogonalmente. Con ambos planteamientos, en los que se han basado la mayoría de las investigaciones realizadas en este ámbito, es difícil poder explicar toda la variación en situaciones de logro, como indican las investigaciones más recientes.

Por ello, el nuevo enfoque de metas de logro 2x2 (Elliot y McGregor, 2001; McGregor y Elliot, 2002) indica que los resultados motivaciones se pueden comprender mejor si se separan las dimensiones en aproximación-evitación, proporcionando evidencia de que cada meta de logro predice un patrón diferente de logro relevante para la maestría y el rendimiento. Pero Papaioannou, Tsigilis, Kosmidou y Milosis (2007) indican que la identificación de prácticas de enseñanza que promueven la evitación-maestría sigue siendo vaga y plantean serias dudas sobre si los estudiantes pueden distinguir entre las prácticas docentes que activan metas de evitación-maestría y las que activan metas de evitaciónrendimiento. De ahí que los dos instrumentos propuestos por Papaioannou et al., (2007) estén basados en el modelo triconómico focalizando las metas en maestríaaproximación, rendimiento-aproximación y rendimientoevitación, utilizado en otras investigaciones (Cury, Elliot, Sarrazin, Da Fonseca y Rufo, 2002; Elliot y Church, 1997). Los alumnos con metas de maestría-aproximación buscan el aprendizaje y el desarrollo personal de habilidades, mientras que en la meta de rendimiento-aproximación tratan de demostrar una mejor ejecución que sus compañeros. La meta de rendimiento-evitación refleja la intención de evitar ser peor que otros.
Cuando Maehr y Nicholls (1980) establecieron el modelo original de metas de logro, la aprobación social fue considerada como una tercera meta de logro, presentando diferentes consecuencias motivacionales sobre las otras dos metas. La describen como la conducta dirigida a maximizar las posibilidades de atribuir gran esfuerzo hacia uno mismo y reducir al mínimo las posibilidades de atribución de bajo esfuerzo hacia uno mismo. Se basan en el supuesto de que el esfuerzo (a diferencia de capacidad) se ve como voluntario, algo que cualquier persona puede mostrar. Por lo tanto, las metas de aprobación social indican compromiso personal en lugar de capacidad o aptitud. Aunque este aspecto ha estado desaparecido de la investigación durante años, algunos investigadores (Papaioannou et al., 2007) han sugerido recientemente que las metas de aprobación social tienen efectos positivos en el logro, siendo necesario examinar las consecuencias del éxito en las relaciones sociales ya que éstas varían de unas culturas a otras.

Según lo expuesto anteriormente, Papaioannou et al. (2007) elaboraron el Achievement Goals Questionnaire (AGQ) que evalúa la metas de logro en las clases de Educación Física en cuatro dimensiones: maestría, aproximaciónrendimiento, evitación-rendimiento y aprobación social, dimensiones que hasta la fecha no recogía ningún instrumento de metas de logro. Los resultados evidencian la validez de criterio y de constructo, en consonancia con el modelo tricotómico de las metas de logro (Elliot, 1997), una aceptable consistencia interna y patrones de correlación entre las dimensiones que apoyan una validez interna de las medidas. Cabe destacar que estos autores también crearon una escala para medir el clima motivacional creado por los profesores de Educación Física siguiendo el principio de compatibilidad (Ajzen y Fishbein, 1977) entre clima del profesor y metas de los alumnos, con la misma estructura que este de metas de logro.

Tras la revisión bibliográfica y hasta donde sabemos, el $A G Q$ en español no ha sido utilizado. La justificación del estudio es paliar este vacío de conocimiento en nuestra lengua, planteándonos como objetivo analizar las propiedades psicométricas de la versión en español del $A G Q$ para determinar fiabilidad y validez de sus puntuaciones, siguiendo la sucesión de análisis de Carretero-Dios y Pérez (2005) referentes a la elaboración de estudios instrumentales. De esta forma, podrá ser utilizado con absoluta garantía en el estudio longitudinal que se está realizando en Costa Rica, México y España sobre aspectos motivacionales en Educación Física y en el tiempo libre. Igualmente, como recogen los propios autores, se pretende adaptar este instrumento a otra cultura con el fin de investigar la generalización transcultural de las consecuencias que se derivan de la investigación del clima motivacional (Papaioannou et al., 2007). Así, se estudia el $A G Q$ en muestras del contexto y cultura costarricense, mexicana y española, sentando las bases para futuras investigaciones de este constructo y sus relaciones con otras variables de interés.

Nuestro trabajo presenta los resultados de dos estudios 
independientes, pero complementarios. En el preliminar, con una muestra piloto de 247 estudiantes, expuesto el proceso de traducción y adaptación de los ítems y mostrados los datos relacionados con las evidencias de validez de contenido de los mismos, se realiza un análisis estadístico de éstos, la exploración de la estructura dimensional de la prueba y un análisis de la fiabilidad del instrumento. En el segundo estudio, con una muestra de 2168 estudiantes de similares características al piloto se analiza, mediante procedimientos confirmatorios, la estructura del instrumento, la validez de constructo, la consistencia interna y la estabilidad temporal en tres países de habla hispana (Costa Rica, México y España).

\section{Estudio 1: estudio psicométrico preliminar del $A G Q$}

\section{Método}

\section{Participantes}

Participaron 247 estudiantes españoles $(126$ chicos $=$ $51 \% ; 121$ chicas $=49 \%$ ) que estudian el primer curso de la Enseñanza Secundaria Obligatoria (ESO) en centros públi$\cos (83 \%)$ y concertados $(17 \%)$. El rango de edad estuvo entre los 11 y los 15 años $(M=12,48 ; D T=.69)$, siendo la edad media en chicos $12.54(D T=.73)$ y $12.42(D T=.65)$ en chicas. Se realizó en enero de 2011.

\section{Instrumento}

Achievement Goals Questionnaire (AGQ) (Papaioannou et al., 2007). El instrumento original contiene 24 ítems para medir las orientaciones de metas de logro de los alumnos en las clases de Educación Física. Se compone de 4 dimensiones: Maestría (ítems 5, 9, 12, 14, 20, 24), Rendimiento-aproximación (ítems 1, 2, 6, 15, 18, 23), Rendimiento-evitación (ítems 4, 7, 10, 13, 16, 21) y Aprobación social (ítems 3, 8, 11, 17, 19, 22). Tiene como encabezado: «En la clase de Educación Física...». Las respuestas se recogen en escala tipo Likert desde 1 (totalmente en desacuerdo) hasta 5 (totalmente de acuerdo). Los valores de consistencia interna de las escalas fueron: Maestría (.82), Rendimiento-aproximación (.87), Rendimiento-evitación (.86) y Aprobación social (.88).

\section{Proceso de adaptación del $A G Q$ en español}

Se aseguró la equivalencia en el proceso de traducción de los ítems. La adaptación española del $A G Q$ (Papaioannou et al., 2007) se realizó atendiendo a los estándares metodológicos internacionales recomendados por la International Test Comision (ITC) para adaptar correctamente test y escalas de unas culturas a otras (Hambleton, 2005; Muñiz y Hambleton, 2000; Muñiz y Bartram, 2007). Con el objetivo de evitar imprecisiones, se combinaron los diseños de traducción directa e inversa de los ítems. Según el procedimiento parallel back-translation (Brislin, 1986), se tradujo la escala del idioma original a la lengua de estudio (inglés a español) por dos traductores bilingües de manera independiente. Las dos traducciones se compararon, se sometieron a discusión ante posibles discrepancias y se obtuvo una versión consensuada de cada ítem. Partiendo de esta versión, la traducción inversa (español a inglés) se llevó a cabo por otros dos traductores bilingües nativos diferentes con amplio conocimiento del idioma objetivo y del idioma fuente y que no conocían la escala original. La bondad de la traducción se juzgó en función del grado de coincidencia con la versión original (Hambleton, 2005), se realizaron modificaciones en aquellos ítems para los que los resultados así lo recomendaban.

La evaluación cualitativa de ítems se efectuó mediante juicio de cuatro expertos, dos en construcción de escalas y dos familiarizados con el constructo a evaluar (Osterlind, 1989). Se les entregó una tabla de especificaciones de ítems (Spaan, 2006), que recogía la definición semántica del constructo y sus componentes. Se mostró el listado de ítems, adaptados de los originales. Debían juzgar su pertinencia y su comprensión en escala de 1 (Muy en desacuerdo) hasta 4 (Muy de acuerdo). Disponían de un apartado para hacer observaciones sobre cada ítem, pudiendo realizar una redacción alternativa si era conveniente. Sólo ítems con puntuaciones medias $<3$, tanto en pertinencia como en comprensión, se revisaron (Otero, González y Calvo, 2012). Si el ítem no se clasificó por al menos 3 de los 4 jueces dentro de las cuatro dimensiones teóricas, se revisó nuevamente, analizando posibles problemas antes de proponer una redacción alternativa que recogiese la dimensión teórica de manera más clara. El encabezamiento fue: «En una escala del 1 (Totalmente en desacuerdo) al 5 (Totalmente de acuerdo), dinos tu grado de desacuerdo o de acuerdo con las siguientes afirmaciones referentes a las clases de Educación Física).

Se administró la versión en español de la escala a 62 escolares españoles cuyos comentarios sobre instrucciones y modo de redacción de ítems condujeron a cambios menores. Tras analizar los resultados psicométricos y una última revisión del equipo de investigación, se llegó a la versión final en español del $A G Q$.

\section{Procedimiento}

Se pidió permiso a los centros educativos mediante carta en la que se explicaban objetivos de investigación y cómo se realizaría, acompañando un modelo del instrumento. Se autoadministró con aplicación masiva, completado anónimamente en una jornada escolar, con consenso y adiestramiento previo de evaluadores. Se informó a los sujetos del objetivo del estudio, la voluntariedad, la absoluta confidencialidad de las respuestas y el manejo de los datos, de que no había respuestas correctas o incorrectas y solicitándoles máxima sinceridad y honestidad. Solamente los alumnos que contaban con el consentimiento informado de los progenitores o y tutores participaron en la investigación. Este trabajo posee informe favorable de la Comisión de Bioética de la Universidad de Murcia. 


\section{Análisis estadísticos}

Se realizó un análisis de los ítems manteniendo la distribución ítem-factor observada en el instrumento original (Papaioannou et al., 2007). Los criterios para conservar un ítem fueron: coeficiente de correlación corregido ítem-total $(C C I T-c) \geq .30$, desviación típica $(D T)>1$ y que todas las opciones de respuesta fueron usadas en algún momento (Nunnally y Bernstein, 1995). Se calculó la fiabilidad de cada componente propuesto originalmente por los autores a través del índice de consistencia interna alfa de Cronbach. Igualmente, se hizo un análisis factorial exploratorio para obtener la estructura factorial interna. Se utilizó el SPSS 17.0.

\section{Resultados}

Análisis de items y homogeneidad

Los ítems de la primera dimensión (Maestría) presentaron valores medios entre 3.93 y 4.23. Las DTs fueron $>1$, estando entre 1.02 y 1.19. La consistencia interna fue aceptable $\left(\alpha_{1}=.78\right)$, siendo todos los CCIT-c $>.41$.

La segunda dimensión (Rendimiento-aproximación) muestra ítems con valores medios entre 2.83 y $3.38, D T s>1$ (oscilando entre 1.32 y 1.46) y todos $\operatorname{los} C C I T-c>.48$. La consistencia interna fue adecuada $\left(\alpha_{2}=.82\right)$.

Los ítems de la tercera dimensión (Rendimiento-evitación) obtuvieron puntuaciones medias entre 2.46 y 3.03 y DTs $>1$ (entre 1.33 y 1.45). Los CCIT-c $>.46\left(\alpha_{3}=.79\right)$.

La cuarta dimensión (Aprobación social) presentó valores medios de respuesta, oscilando entre 2.60 y 4.09. Las DTs fueron $>1$, estando entre 1.04 y 1.40 . La consistencia interna fue alta $\left(\alpha_{4}=.79\right)$, siendo todos CCIT $-c>.41$.

Por tanto, ningún ítem de las cuatro dimensiones se eliminó al cumplir los requisitos de $D T>1$, correlación ítemtotal $>.30$ y fiabilidad aceptable en cada dimensión (entre .78 y .82) (Tabla 1).

Tabla 1. Número de sujetos (n), media (M), desviación típica (DT), asimetría, curtosis, correlación ítem-total (CCIT-c), alfa de la escala teórica y la dimensión si algún ítem es eliminado ( $\alpha$ sin ítem) del AGQ.

\begin{tabular}{|c|c|c|c|c|c|c|c|}
\hline & $n$ & $M$ & $D T$ & $\begin{array}{l}\text { Asime- } \\
\text { tría }\end{array}$ & $\begin{array}{l}\text { Cur- } \\
\text { tosis }\end{array}$ & $C C I T-c$ & $\begin{array}{l}\alpha \sin \\
\text { ítem }\end{array}$ \\
\hline \multicolumn{8}{|l|}{ Maestría $\left(\alpha_{1}=.782\right)$} \\
\hline 5.Mi meta constantemente es desarrollar mis habilidades & 240 & 4.03 & 1.03 & -.823 & .01 & .45 & .70 \\
\hline 9.Trato de participar y/o esforzarme más con el fin de aprender nuevas habilidades y juegos & 243 & 4.19 & 1.05 & -1.11 & .74 & .50 & .68 \\
\hline 12.Es importante para mí aprender nuevas cosas siempre & 242 & 4.23 & 1.02 & -1.30 & .73 & .44 & .70 \\
\hline 14.Cuando aprendo una habilidad quiero repetirla más veces & 240 & 3.95 & 1.18 & -1.01 & .21 & .45 & .70 \\
\hline 20.Me gusta aprender nuevas habilidades sin importar la dificultad de las mismas & 242 & 3.93 & 1.19 & -1.08 & .37 & .54 & .67 \\
\hline $\begin{array}{l}\text { 24. Me gusta hacer un esfuerzo en ejercicios difíciles porque este es el camino para desarrollar? } \\
\text { mis habilidades }\end{array}$ & & 4.08 & 1.05 & -1.08 & .66 & .41 & .70 \\
\hline \multicolumn{8}{|l|}{ Rendimiento-aproximación $\left(\alpha_{2}=.823\right)$} \\
\hline 1.Me siento muy bien cuando soy el único que puede hacer un ejercicio & 243 & 3.25 & 1.40 & -.19 & -1.16 & .54 & .80 \\
\hline 2.Estoy completamente satisfecho cuando supero a los demás & 243 & 3.32 & 1.32 & -.35 & -.98 & .69 & .77 \\
\hline 6.Siento éxito cuando soy el único que puede hacer una habilidad o juego & 243 & 3.38 & 1.34 & -.38 & -.96 & .71 & .77 \\
\hline 15.Trato siempre de intentar llevar la delantera a los demás en habilidades o juegos & 242 & 2.88 & 1.37 & .05 & -1.17 & .52 & .81 \\
\hline 18.Siempre intentaré superar a mis compañeros de clase & 242 & 2.83 & 1.46 & .10 & -1.36 & .61 & .79 \\
\hline 23.Estoy satisfecho cuando los demás no pueden hacer algo tan bien como yo lo hago & 242 & 2.88 & 1.45 & .06 & -1.32 & .48 & .82 \\
\hline \multicolumn{8}{|l|}{ Rendimiento-evitación $\left(\alpha_{3}=.786\right)$} \\
\hline 4.Procuro evitar habilidades o juegos por los que se puedan burlar de mí & 243 & 2.65 & 1.44 & .29 & -1.23 & .46 & .77 \\
\hline 7.Frecuentemente me preocupa que me digan que no tengo ninguna habilidad & 242 & 3.03 & 1.45 & -.08 & -1.30 & .52 & .76 \\
\hline 10.Evito habilidades y juegos en los que no soy capaz de hacerlo bien & 242 & 2.56 & 1.34 & .35 & -1.04 & .56 & .75 \\
\hline 13.Me preocupa aparentar que no soy capaz de hacerlo bien & 240 & 2.95 & 1.38 & -.04 & -1.25 & .55 & .75 \\
\hline $\begin{array}{l}\text { 16.Frecuentemente me preocupa que si intento hacer una actividad pueda parecer que no soy? } \\
\text { capaz de hacerlo bien }\end{array}$ & y 242 & 2.83 & 1.33 & -.02 & -1.12 & .58 & .74 \\
\hline 21.Suelo evitar ejercicios y juegos en los que puedo parecer incapaz & 241 & 2.46 & 1.43 & .51 & -1.07 & .55 & .75 \\
\hline \multicolumn{8}{|l|}{ Aprobación social $\left(\alpha_{4}=.785\right)$} \\
\hline 3.Es importante para mí aprender un juego o habilidad para que los demás me quieran & 243 & 2.60 & 1.33 & .29 & -1.03 & .53 & .75 \\
\hline 8.Estoy contento cuando desarrollo mis habilidades y los demás me valoran & 243 & 4.09 & 1.04 & -1.22 & 1.12 & .41 & .80 \\
\hline 11.Me gusta aprender nuevas habilidades y juegos para ganarme la aprobación de los demás & 242 & 3.34 & 1.27 & -.40 & -.75 & .60 & .74 \\
\hline 17.Quiero alcanzar un rendimiento alto para que los demás otros me quieran & 239 & 2.81 & 1.37 & .06 & -1.16 & .65 & .72 \\
\hline 19.Me gusta hacer un ejercicio perfectamente para sentirme querido por los demás & 242 & 2.84 & 1.40 & .11 & -1.25 & .61 & .73 \\
\hline $\begin{array}{l}\text { 22.Me siento absolutamente exitoso cuando hago algo bien y los demás me den el vist } \\
\text { bueno }\end{array}$ & 242 & 3.62 & 1.24 & -.61 & -.54 & .50 & .76 \\
\hline
\end{tabular}

Se realizó un análisis de correlación entre las puntuaciones de los ítems y las puntuaciones totales en cada uno de los componentes. Ningún ítem mostró solapamiento entre las cuatro dimensiones teóricas, por lo que cada ítem solamente obtuvo una alta correlación con su dimensión de pertenencia. Se siguieron las indicaciones realizadas por Carrete- 
ro-Dios y Pérez (2005) para tratar de configurar componentes lo más homogéneos posibles. Así, no se descartó ningún ítem, ya que las diferencias entre la correlación observada para ese ítem y el total en su dimensión y la apreciada para ese mismo ítem y las puntuaciones totales en las otras cuatro dimensiones fueron positivas, a favor de la dimensión teórica de pertenencia en al menos .20 (Jackson, 1970).

\section{Estructura y consistencia interna}

Los índices de asimetría y curtosis fueron próximos a cero y $<2.0$ (Bollen y Long, 1994). La medida de adecuación muestral de Kaiser-Meyer-Olkin $(K M O=.84)$ fue aceptable y el test de Bartlett resultó estadísticamente significativo $\left(\chi^{2}(276)=1960.05, p<.000\right)$. Se calculó mediante el método de extracción de máxima verosimilitud y rotación de normalización oblicua. Los resultados confirman la extracción de cuatro factores: Maestría, Rendimiento-aproximación, Rendimientoevitación y Aprobación social (Tabla 2).

$\mathrm{El}$ análisis de correlación indicó que la dimensión Maestría tenía una baja correlación con Rendimiento-aproximación ( $r$ $=.20)$ y no correlacionaba con Rendimiento-evitación $(r=.006)$, mientras que estas dos dimensiones presentaban una moderada correlación $(r=.40)$. La dimensión Aprobación social convergía positivamente con todas las demás dimensiones, con valores entre .31 y .67 .

Por lo tanto, la versión final española del $A G Q$ quedó compuesta, como la original, por cuatro dimensiones con un total de 24 ítems (Anexo 1) que hacen referencia a: Maestría (ítems 5, 9, 12, 14, 20, 24), Rendimiento-aproximación (ítems 1, 2, 6, 15, 18, 23), Rendimiento-evitación (ítems 4, 7, 10, 13, 16, 21) y Aprobación social (ítems 3, 8, 11, 17, 19, 22). Las respuestas se recogen con escala tipo Likert desde 1 (totalmente en desacuerdo) hasta 5 (totalmente de acuerdo).

Tabla 2. Estructura factorial rotada, comunalidades, autovalores, alfa de Cronbach y porcentaje de varianza explicada por cada dimensión $(N=247)$ del AGQ.

\begin{tabular}{|c|c|c|c|c|c|}
\hline Factor & F1 & $\mathrm{F} 2$ & F3 & F4 & $\mathrm{h}^{2}$ \\
\hline 5.Mi meta constantemente es desarrollar mis habilidades & & & & .67 & .48 \\
\hline 9.Trato de participar y/o esforzarme más con el fin de aprender nuevas habilidades y juegos & & & & .83 & .68 \\
\hline 12.Es importante para mí aprender nuevas cosas siempre & & & & .76 & .54 \\
\hline 14.Cuando aprendo una habilidad quiero repetirla más veces & & & & .73 & .54 \\
\hline 20.Me gusta aprender nuevas habilidades sin importar la dificultad de las mismas & & & & .78 & .65 \\
\hline 24.Me gusta hacer un esfuerzo en ejercicios difíciles porque este es el camino para desarrollar mis habilidades & & & & .62 & .58 \\
\hline 1.Me siento muy bien cuando soy el único que puede hacer un ejercicio & & & .89 & & .75 \\
\hline 2.Estoy completamente satisfecho cuando supero a los demás & & & .86 & & .80 \\
\hline 6.Siento éxito cuando soy el único que puede hacer una habilidad o juego & & & .79 & & .79 \\
\hline 15.Trato siempre de intentar llevar la delantera a los demás en habilidades o juegos & & & .66 & & .52 \\
\hline 18.Siempre intentaré superar a mis compañeros de clase & & & .75 & & .66 \\
\hline 23.Estoy satisfecho cuando los demás no pueden hacer algo tan bien como yo lo hago & & & .56 & & .54 \\
\hline 4.Procuro evitar habilidades o juegos por los que se puedan burlar de mí & & .69 & & & .50 \\
\hline 7.Frecuentemente me preocupa que me digan que no tengo ninguna habilidad & & .78 & & & .60 \\
\hline 10.Evito habilidades y juegos en los que no soy capaz de hacerlo bien & & .83 & & & .65 \\
\hline 13.Me preocupa aparentar que no soy capaz de hacerlo bien & & .75 & & & .57 \\
\hline $\begin{array}{l}\text { 16. Frecuentemente me preocupa que si intento hacer una actividad pueda parecer que no soy capaz de hacerlo } \\
\text { bien }\end{array}$ & & .79 & & & .62 \\
\hline 21.Suelo evitar ejercicios y juegos en los que puedo parecer incapaz & & .75 & & & .69 \\
\hline 3.Es importante para mí aprender un juego o habilidad para que los demás me quieran & .63 & & & & .41 \\
\hline 8.Estoy contento cuando desarrollo mis habilidades y los demás me valoran & .51 & & & & .41 \\
\hline 11.Me gusta aprender nuevas habilidades y juegos para ganarme la aprobación de los demás & .60 & & & & .60 \\
\hline 17.Quiero alcanzar un rendimiento alto para que los demás otros me quieran & .83 & & & & .72 \\
\hline 19.Me gusta hacer un ejercicio perfectamente para sentirme querido por los demás & .786 & & & & .71 \\
\hline 22.Me siento absolutamente exitoso cuando hago algo bien y los demás me den el visto bueno & .55 & & & & .51 \\
\hline$\%$ Varianza explicada & \multicolumn{5}{|c|}{5.259 .2113 .7026 .6454 .80} \\
\hline Autovalor & \multicolumn{5}{|c|}{1.462 .153 .326 .46} \\
\hline Kaiser-Meyer-Olkin (KMO) & \multicolumn{5}{|l|}{.84} \\
\hline Test esfericidad Bartlett & \multicolumn{5}{|c|}{$\chi_{(276)}^{2}=1960.05, p<.000$} \\
\hline Alfa Cronbach & \multicolumn{5}{|c|}{$.78 \quad .82 \quad .79$} \\
\hline
\end{tabular}

Nota: los ítems son listados en orden decreciente según su saturación en el factor 1. Se incluyen valores de saturaciones superiores a .50.

$\mathrm{F} 1=$ Maestría $. \mathrm{F} 2=$ Rendimiento-aproximación. $\mathrm{F} 3=$ Rendimiento-evitación $. \mathrm{F} 4=$ Aprobación social. 
Estudio 2: análisis factorial confirmatorio, validez de constructo, consistencia interna y estabilidad temporal

\section{Método}

\section{Participantes}

Participaron 2168 estudiantes del primer curso de Enseñanza Secundaria (cursos equivalentes en los tres países), seleccionados aleatoriamente para participar en un estudio longitudinal, de Costa Rica (423), México (408) y España (1337), siendo 1052 chicos (50.40\%), 1037 chicas (49.60\%) y 79 no reflejaron el sexo, de centros públicos $(86.60 \%)$ y concertados $(13.40 \%)$. El rango de edad estuvo entre los 11 y los 16 años $(M=12.49 ; D T=.81)$, siendo la edad media en chicos $12.53(D T=.87)$ y $12,44(D T=.74)$ en chicas. Se realizó entre febrero-junio de 2011. Las muestras fueron equivalentes en los tres países ya que no existen diferencias estadísticamente significativas por sexo $(p=.47)$, edad $(p=$ .56) y tipo de centro $(p=.46)$.

Para evaluar la estabilidad temporal del $A G Q$ se utilizó una segunda muestra de 140 alumnos, previamente elegida aleatoriamente $(72$ chicos y 68 chicas) perteneciente a los tres países $\left(M_{\text {edad }}=12.53\right.$ años; $\left.D T=.85\right)$, que completó nuevamente el instrumento cuatro semanas después. Para prevenir posibles tendencias de deseabilidad social, los sujetos se identificaron a través de su fecha de nacimiento.

\section{Instrumentos}

Se administró la versión en español del Acbievement Goals Questionnaire (AGQ) (Papaioannou et al., 2007), derivada del estudio 1 (Anexo 1).

\section{Procedimiento}

Se utilizó el mismo procedimiento del estudio 1: permiso a centros, voluntariedad, autoadministrado con aplicación masiva, presencia de encuestador, completado anónimamente en jornada escolar, con consenso y adiestramiento previo de los evaluadores, con consentimiento informado de progenitores y tutores.

\section{Análisis estadísticos}

Se aplicaron modelos de ecuaciones estructurales para confirmar la dimensionalidad propuesta teóricamente ( $\mathrm{Pa}$ paioannou et al., 2007). La estructura factorial del $A G Q$ se evaluó con $A F C$, con AMOS 7.0, utilizando el método de estimación de máxima verosimilitud, procedimiento de estimación de modelos de ecuaciones estructurales que asume una distribución normal de los datos y una escala continua de ellos.

Lo más adecuado para aceptar o rechazar un modelo es emplear combinación de varios índices (Bentler, 1995). El ajuste del modelo se evaluó con una combinación de índices de ajustes absolutos y relativos. Entre los absolutos, se utilizaron valor $p$, ratio entre chi-cuadrado y grados de libertad $\left(\chi^{2} / g l\right)$. En un modelo considerado perfecto su valor sería 1,0 y las ratios $<2$ se considerarán indicadores de muy buen ajuste del modelo, mientras que valores $<5$ son considerados aceptables. Sin embargo, estos índices están afectados por el tamaño muestral, por lo que se utilizó el índice SRMR en el que valores $\leq .06$ indican un excelente ajuste y valores $\leq .08$ un buen ajuste (Hu y Bentler, 1999).

De los índices relativos, se seleccionó el IFI y el CFI porque su rango de bondad se sitúa entre 0 y 1 y es más fácil de interpretar que otros índices de ajuste e indica reducciones en ajustes pobres (Hoyle, 1995). También se utilizó el TLI. En los índices incrementales (CFI y TLI) sus valores aceptables son $>.85$ (Schumacker y Lomax, 1996), aunque lo ideal son valores $\geq .90 \mathrm{y}>.95$ que señalarían un ajuste excelente (Kline, 1998). Browne y Cudeck (1993) recomiendan utilizar el RMSEA y, según Jöreskog y Sörbom (1993), el modelo basado en la muestra utilizada representa excelentemente a la población si su valor es $\leq .05$, considerándose un ajuste aceptable cuando es $<.08$.

Se realizaron con SPSS 17.0 todos los análisis: análisis de ítems, homogeneidad y estructura interna, correlación (coeficiente de Pearson), consistencia interna (alfa de Cronbach), estabilidad temporal a partir de la correlación test-retest. Se realizó un análisis para estimar las diferencias de sexo en cada país, según las puntuaciones medias, en las cuatro dimensiones mediante el $t$-test, teniendo en cuenta el test de Levene para estimar la igualdad de las varianzas con un nivel de significación de $p<.01$. Se calculó el tamaño del efecto para ver la relevancia de las diferencias encontradas, utilizando los valores de la media y la desviación típica $\left(d=\left(\mathrm{X}_{1}-\mathrm{X}_{2}\right) / \sigma\right)$. Se puede considerar grande o pequeño un determinado valor según estas orientaciones (Cohen, 1988): $d=.20$ (pequeño), $d=$ .50 (moderado) y $d=.80$ (grande).

\section{Resultados}

\section{Descripción inicial de los datos}

En los tres países, los índices de asimetría y curtosis fueron próximos a cero y $<2.0$ (Bollen y Long, 1994), lo que indica semejanza con la curva normal univariada y permite utilizar técnicas factoriales de máxima verosimilitud en el $A F C$. Los datos descriptivos $(M, D T)$ fueron similares al estudio 1 .

Los índices de correlación ítem-total corregidos son adecuados, ya que todos fueron $\geq .40$ en las cuatro dimensiones (Maestría, Rendimiento-aproximación, Rendimiento-evitación y Aprobación social) y en la muestra de los tres países, al igual que la correlación entre la puntuación de los ítems y la puntuación total en las cuatro dimensiones, que fueron siempre positivas a favor de la dimensión teórica de pertenencia en al menos .20 (Jackson, 1970). 


\section{Análisis factorial confirmatorio}

El modelo puesto a prueba hipotetiza la existencia de cuatro variables latentes independientes Maestría, Rendimientoaproximación, Rendimiento-evitación y Aprobación social, que subyacen a las 24 variables observables y dan cuenta de las covarianzas observadas entre ellas. Los resultados mostraron adecuados índices de bondad de ajuste (Tabla 3) y coeficientes estandarizados tanto en Costa Rica, México como en España (Figura 1).

Tabla 3. Índices de ajuste del modelo del AGQ por países.

\begin{tabular}{lllllll}
\hline & $\chi^{2} / g l$ & IFI & TLI & CFI & RMSEA & SRMR \\
\hline Costa Rica & 3,37 & .91 & .90 & .91 & .035 & .044 \\
México & 2,96 & .91 & .90 & .91 & .044 & .048 \\
España & 2,99 & .95 & .95 & .94 & .031 & .034 \\
\hline Deseable & $<3$ & $>.90$ & $>.90$ & $>.90$ & $<.05$ & $<.06$ \\
\hline
\end{tabular}

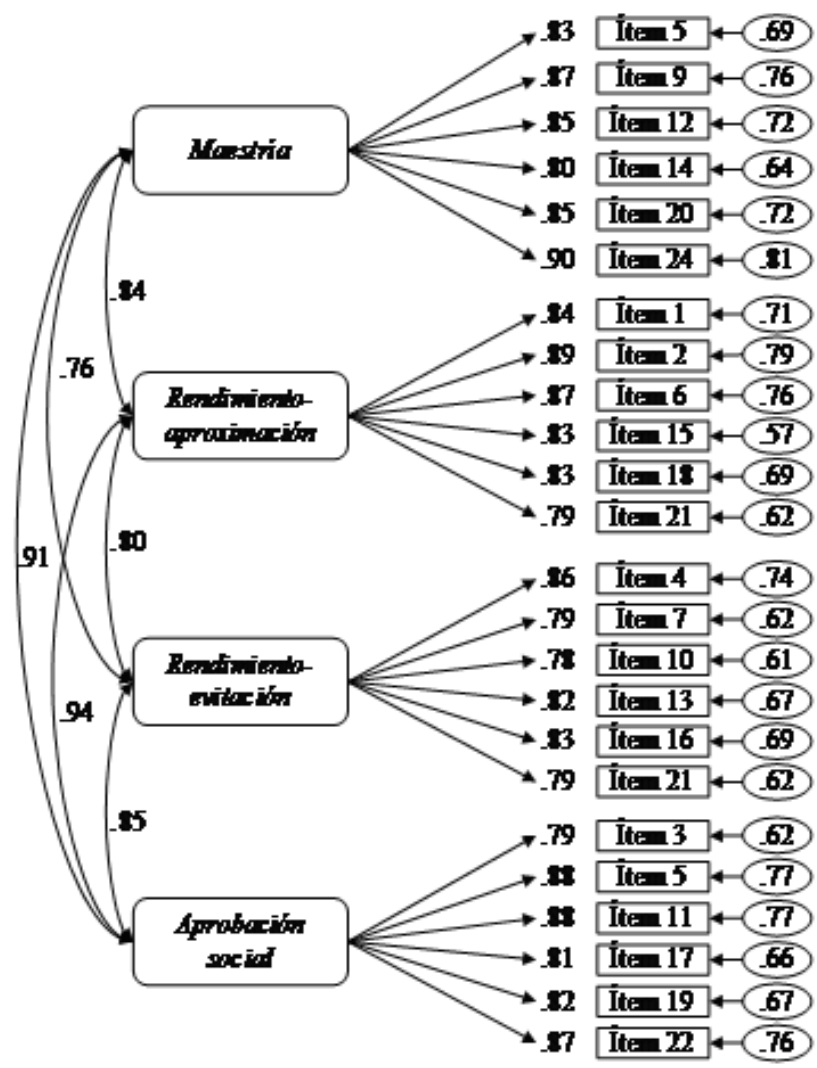

Figura 1. Path Diagram del análisis factorial confirmatorio, con pesos estandarizados y errores de medición de cada uno de los ítems del AGQ versión en español, en la muestra de estudiantes españoles.

\section{Análisis de fiabilidad}

La consistencia interna de las cuatro dimensiones se evaluó con el alfa de Cronbach (Tabla 4). Los valores se situa- ron entre .73 (Maestría) y .83 (Rendimiento-evitación).

La estabilidad temporal se evaluó con muestra de 140 estudiantes que completaron el $A G Q$ en dos ocasiones, con intervalo de cuatro semanas. Los resultados de consistencia interna del pretest oscilaron entre .73 y .83 y los resultados del postest entre .74 y .83, situándose los valores de la correlación test-retest entre .81 y .83 (Tabla 4).

Tabla 4. Consistencia interna y correlaciones test-retest de las cuatro dimensiones del AGQ.

\begin{tabular}{|c|c|c|c|c|c|}
\hline & Alfa & Alfa & Alfa & Correlaciones \\
\hline & & \multicolumn{4}{|c|}{ muestra pretest postest test-retest } \\
\hline \multirow{5}{*}{$\begin{array}{l}\text { Costa } \\
\text { Rica }\end{array}$} & $n$ & 423 & 30 & 30 & 30 \\
\hline & Maestría & .80 & .81 & .82 & .83 \\
\hline & $\begin{array}{l}\text { Rendimiento- } \\
\text { aproximación }\end{array}$ & .82 & .81 & .81 & .82 \\
\hline & $\begin{array}{l}\text { Rendimiento- } \\
\text { evitación }\end{array}$ & .83 & .83 & .83 & .83 \\
\hline & $\begin{array}{l}\text { Aprobación } \\
\text { social }\end{array}$ & .78 & .80 & .79 & .81 \\
\hline \multirow{5}{*}{ Méxice } & $n$ & 408 & 35 & 35 & 35 \\
\hline & Maestría & .73 & .73 & .74 & .81 \\
\hline & $\begin{array}{l}\text { Rendimiento- } \\
\text { aproximación }\end{array}$ & .79 & .79 & .80 & .82 \\
\hline & $\begin{array}{l}\text { Rendimiento- } \\
\text { evitación }\end{array}$ & .76 & .77 & .76 & .81 \\
\hline & $\begin{array}{l}\text { Aprobación } \\
\text { social }\end{array}$ & .78 & .79 & .78 & .81 \\
\hline \multirow{5}{*}{ Españ } & $n$ & 1337 & 85 & 85 & 85 \\
\hline & Maestría & .76 & .78 & .77 & .82 \\
\hline & $\begin{array}{l}\text { Rendimiento- } \\
\text { aproximación }\end{array}$ & .83 & .82 & .81 & .82 \\
\hline & $\begin{array}{l}\text { Rendimiento- } \\
\text { evitación }\end{array}$ & .77 & .78 & .79 & .83 \\
\hline & $\begin{array}{l}\text { Aprobación } \\
\text { social }\end{array}$ & .80 & .81 & .81 & .82 \\
\hline
\end{tabular}

\section{Correlaciones entre dimensiones}

Las correlaciones entre las cuatro dimensiones del $A G Q$ (coeficiente de Pearson) utilizadas para evaluar la validez de constructo y la presencia de formas específicas de asociación, indican que la dimensión Maestría tiene una baja correlación con Rendimiento-aproximación y no correlaciona con Rendimiento-evitación, mientras que estas dos dimensiones presentan una correlación moderada. La dimensión Aprobación social converge positivamente con todas las demás dimensiones, con valores entre moderados y altos, entre .35 y .66 (Tabla 5). 
$\underline{\text { Tabla 5. Correlaciones entre las cuatro dimensiones del AGQ }}$

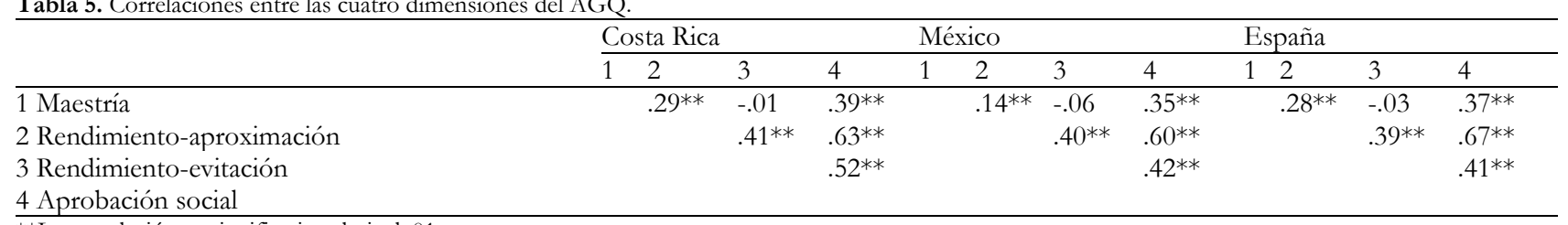

**La correlación es significativa al nivel .01.

\section{Diferencias de sexo porpaises}

Los resultados sobre las orientaciones de meta de logro que tienen los alumnos de sus clases de Educación Física son bastante similares en Costa Rica, México y España. Las medias mayores se obtienen en la dimensión Maestría, encontrándose diferencias estadísticamente significativas, en los tres países, siendo la puntuación en chicos más alta que en chicas (tamaño del efecto entre pequeño y moderado), al igual que sucede con las dimensiones Rendimiento-aproximación y Aprobación social. En la dimensión Rendimiento-evitación, en los tres países, no existen diferencias estadísticamente significativas (Tabla 6).

Tabla 6. Medias $(M)$, desviaciones típicas $(D T)$, significación ( $p$ valor) y tamaño del efecto $(d)$ del AGQ, diferencias por sexo y países.

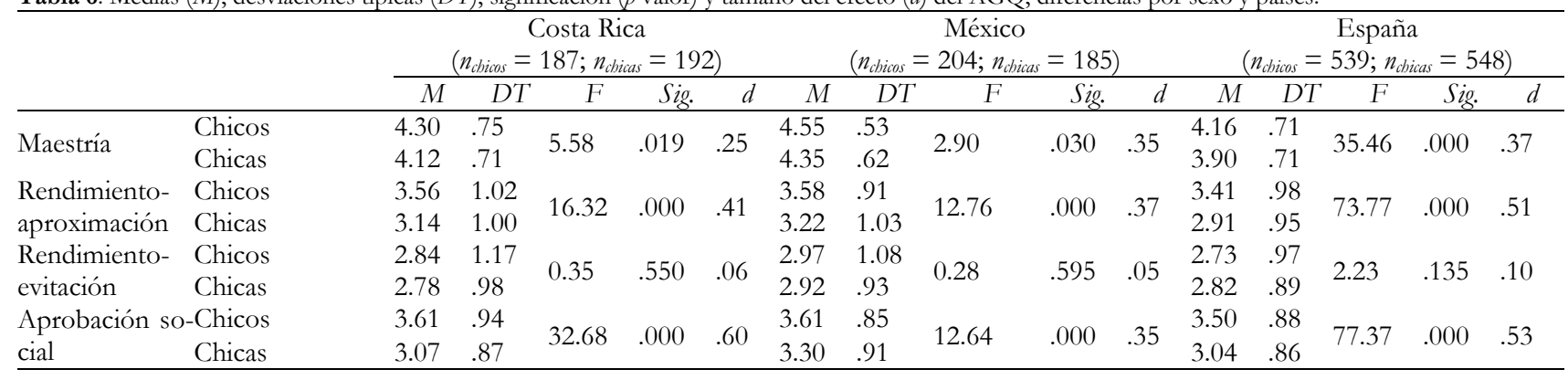

\section{Discusión y conclusiones}

Las orientaciones de meta presentan una considerable importancia por sus relaciones positivas con la participación en actividades físico-deportivas y con distintos aspectos de la motivación, en particular, la motivación intrínseca y sus implicaciones pedagógicas (Amado, Leo, Sánchez, González y López, 2012; Díaz y Aguado, 2012; Duda y Nicholls, 1992; Flores, Salguero y Márquez, 2008; Papaioannou y McDonald, 1993; Peiró y Sanchís, 2004; Sánchez et al., 2009, Walling y Duda, 1995, Wang et al., 2008). Hasta la fecha no existe, en español, un instrumento de metas de logro en Educación Física con estas cuatro dimensiones dentro del modelo tricotómico: maestría, aproximación-rendimiento, evitaciónrendimiento y aprobación social.

Uno de los objetivos conseguidos en este trabajo instrumental es validar el $A G Q$, en español, en tres contextos diferentes (Costa Rica, México y España). Los análisis psicométricos realizados así lo corroboran. Al igual que en la versión original, se encontró la reproducción de la estructura factorial de cuatro dimensiones (Maestría, Aproximación-rendimiento, Evitación-rendimiento y Aprobación social) del modelo original que se hipotetizaba (Papaioannou et al., 2007), que evalúa las metas de logro de los alumnos en las clases de Educación Física. Además, se apreció que las saturaciones de cada uno de los ítems son aceptables (en los tres países), lo que confirma la estructura de cuatro factores con seis ítems en cada uno de ellos. Estos resultados encuentran apoyo transcultural con alumnado costarricense, mexicano y español, utilizando la traducción al español del $A G Q$, además con una amplia muestra.

Por otra parte, la asociación de las dimensiones Rendimiento-aproximación y Rendimiento-evitación, al igual que sucedió en la versión original, es moderadamente positiva lo que está en consonancia con el modelo tricotómico de metas de logro (Elliot, 1997). Sin embargo, la asociación de Maestría con Rendimiento-aproximación es baja y positiva, a diferencia de la versión original en la que no correlacionaba. Igualmente, Maestría no estuvo correlacionada con Rendimiento-evitación, que fue baja y negativa originalmente. Estos resultados no apoyan la validez interna del instrumento original por lo que sería necesario realizar más investigaciones con este instrumento para verificar este aspecto.

La dimensión Aprobación social está vinculada positivamente, como era de esperar, con las demás dimensiones de rendimiento, presentando unos valores de correlación ligeramente superiores a la versión original. Estos resultados apoyan la validez interna ya que una característica común de la Aprobación social y de las metas de rendimiento son unos logros basados en criterios determinados por otros. Cuando se persiguen metas de rendimiento, los logros de uno se juzgan en relación con el logro de los demás. Cuando se adoptan metas sociales de aprobación el propio logro se juzga en función de si los demás aprueban la conducta del sujeto ( $\mathrm{Pa}$ paioannou et al., 2007). En resumen, estos patrones de co- 
rrelación entre maestría, rendimiento y aprobación social evidencian la estructura interna de las medidas.

Igualmente, la consistencia interna es bastante aceptable, se encontró dentro de los valores alcanzados en la investigación de Papaioannou et al. (2007). Además, todos los ítems tienen una correlación elevada con su factor correspondiente y baja con los del otro, por tanto, no ha sido necesario eliminar ninguno.

Las orientaciones de meta que tienen los alumnos en las clases de Educación Física son bastante similares en Costa Rica, México y España. La predominancia de la Maestría predice consecuencias favorables sobre la participación en actividades físicas y deportivas (White y Duda, 1994) y, sobre todo, para la persistencia y mantenimiento de la actividad a lo largo de los años (Papaioannou, Bebetsos, Theodorakis, Christodoulidis y Kouli, 2006). Los individuos orientados a la Maestría tienden a atender para aprender nuevas habilidades, esforzarse y demostrar control del comportamiento. Esta orientación crea un desarrollo personal y refuerza el compromiso para participar en deportes organizados, empleando el tiempo libre de forma activa y saludable (Amado et al., 2012; Ruiz-Juan et al., 2011).

Los análisis de varianza realizados para ver si existían diferencias significativas según el sexo, indican que los chicos presentaban mayor Rendimiento-aproximación y Aprobación social en comparación con las chicas, resultado consistente con estudios previos sobre las diferencias por sexo en Educación Física (Goudas y Biddle, 1994; Pozo y Tejada, 2012; Van Der Horst, Paw, Twisk y Van Mechelen, 2007). Esto nos viene a indicar que ellos consideran la Educación Física como un medio para adquirir un cierto status de reconocimiento social, al igual que hacer una demostración hacia el exterior de que son mejores y superiores que sus iguales. Efecti-

\section{Referencias}

Ajzen, I. y Fishbein, M. (1977). Attitude-behavior relations: A theoretical analysis and review of empirical research. Psychological Bulletin, 84(5), 888918.

Amado, D., Leo, F., Sánchez, D., González, I. y López, J. (2012). It is compatible the sport in scholar age with other social roles? A study through Self-Determination Theory. Retos, 21, 50-52.

Ames, C. (1992). Classrooms: Goals, structures, and student motivation. Journal of Educational Psychology, 84, 261-271.

Ames, C. y Archer, J. (1988). Achievement goals in the classroom: Students' learning strategies and motivation processes. Journal of Educational Psychology, 80, 260-267.

Balaguer, I., Castillo, I., Duda, J. y García-Merita, M. (2011). Asociaciones entre la percepción del clima creado por el entrenador, orientaciones disposicionales de meta, regulaciones motivacionales y vitalidad subjetiva en jóvenes jugadoras de tenis. Revista de Psicología del Deporte. 20, 133148.

Bentler, P. M. (1995). EQS structural equations program manual. Encino, C.A: Multivariate Software.

Bollen, K. A. y Long, J. S. (1994). Testing structural equation models. Newbury Park, CA: Sage.

Brislin, R. W. (1986). The wording and translation of research instruments. En W. Lonner y J. Berry (Eds.), Field methods in cross-cultural research (pp. 137-164). Beverly Hills, CA: Sage.

Browne, M. y Cudeck, R. (1993). Alternative ways of assessing model fit. En K. Bollen y J. Long (Eds.), Testing structural equation models (pp. 136-162). vamente, el rechazo de las chicas al ego es coherente con los datos de la literatura internacional, también en jóvenes deportistas. En un estudio en jóvenes europeos deportistas (Ledent et al., 1996), en todos los países, chicos y chicas desarrollaron una orientación a la tarea más fuerte que la orientación al ego, al igual que sucede en nuestro estudio, algo que se observa también en otro trabajo en jóvenes mexicanos (Ruiz-Juan y Piéron, 2013).

Podemos concluir que los análisis psicométricos del AGQ demuestran que las puntuaciones obtenidas al aplicar el cuestionario resultarán válidas y fiables y que viene a ocupar el vacío de conocimiento existente al respecto en diferentes contextos de habla hispana. Además, se confirma la existencia de las cuatro dimensiones que se hipotetizan desde el modelo teórico de partida. Destacar la gran utilidad de esta versión en futuras investigaciones para valorar las metas de logro de los alumnos en las clases de Educación Física. Aun así, estimamos importante efectuar más estudios con diferentes muestras, para seguir obteniendo más evidencias de diferentes fuentes de su validez. Así se hará en el estudio longitudinal que se está llevando a cabo en los tres países reseñados.

Para investigaciones futuras queda abierto un vasto campo de posibilidades de investigación, como sugiere la literatura consultada. Además de aplicar el $A G Q$, es conveniente validar en español la escala creada por Papaioannou et al. (2007) para medir el clima motivacional originado por el profesor de Educación Física y verificar también que se cumple el principio de compatibilidad entre clima motivacional del profesor y metas de los alumnos, al igual que ver la relación que guarda con otros aspectos psicológicos como motivación y satisfacción, lo cual aportaría nuevas y valiosas evidencias sobre la validez del $A G Q$.

Thousand Oaks: Sage.

Carretero-Dios, H. y Pérez, C. (2005). Normas para el desarrollo y revisión de estudios instrumentales. International Journal of Clinical and Health Psychology, 5, 521-551.

Cohen, J. (1988). Statistical Power Analysis for the Behavioral Sciences. 2nd. edit., Hillsdale, N. J., Erlbaum (primera edición, 1977 New York: Academic Press).

Cury, F., Elliot, A. J., Sarrazin, P., Da Fonseca, D. y Rufo, M. (2002). The trichotomous achievement goal model and intrinsic motivation: A sequential analysis. Journal of Experimental Social Psychology, 38, 473-481.

Díaz, M. y Aguado, R. (2012). Perceived competence of experienced physical education teachers about the task as a didactic resource. Retos, 22, 16-18.

Duda, J. L. y Nicholls, J. G. (1992). Dimensions of achievement-motivation in schoolwork and sport. Journal of Educational Psychology, 84, 290-299.

Duda, J. L. y Whitehead, J. (1998). Measurement of goal perspectives in the physical domain. In J. Duda (Ed.), Advances in sport and exercise psychology measurement (pp. 21-48). Morgantown, WV: Fitness Information Technology.

Dweck, C. S. (1986). Motivational processes affecting learning. The American Psychologist, 41, 1040-1048.

Elliot, A. J. (1997). Integrating the "classic" and "contemporary" approaches to achievement motivation: A hierarchical model of approach and avoidance achievement motivation. In M. Maehr y P. Printrich (eds.), Advances in motivation and achievement (Vol. 10) (pp. 243-279). Greenwich, 
CT: JAI Press.

Elliot, A. J. (2005). A conceptual history of the achievement goal construct. In A. Elliot y C. Dweck (Eds.), Handbook of competence and motivation (pp. 52-72). New York: Guilford Press.

Elliot, A. J. y Church, M. A. (1997). A hierarchical model of approach and avoidance achievement motivation. Journal of Personality and Social Psychology, 72, 218-232.

Elliot, A. J. y McGregor, H. A. (2001). A 2 X 2 achievement goal framework. Journal of Personality and Social Psychology, 80, 501-519.

Flores, J., Salguero, A. y Márquez, S. (2008). Goal orientations and perceptions of the motivational climate in physical education classes among Colombian students. Teaching and Teacher Education, 24, 1441-1449.

Goudas, M. y Biddle, S. (1994). Perceived motivational climate and intrinsic motivation in school physical education classes. European Journal of Psychology of Education, 9, 241-250.

Hambleton, R. K. (2005). Issues, designs and technical guidelines for adapting tests into multiple languages and cultures. En R. K. Hambleton, P. F. Merenda y S. D. Spielberger (Eds.), Adapting educational and psychological tests for cross-cultural assessment (pp. 3-38). Mahwah, NJ: Lawrence Erlbaum Associates.

Hoyle, R. H. (1995). Structural equation modeling: Concepts, issues, and applications. Thousand Oaks, CA: Sage.

Hu, L. y Bentler, P. M. (1999). Cutoff criteria for fit indexes in covariance structure analysis: Conventional criteria versus new alternatives. Structural Equation Modeling, 6, 1-55.

Jackson, D. N. (1970). A sequential system for personality scale development. En C. D. Spielberger (Ed.), Current topics in clinical and community psychology (pp. 61-96). Nueva York: Academic Press.

Jöreskog, K. G. y Sörbom, D. (1993). Structural equation modeling with the SIMPLIS command language. Chicago: Scientific Software International.

Kline, R. B. (1998). Principles and practice of structural equation modeling. New York: Guilford.

Ledent, M., Telama, R., Cloes, M., Carvalho, L. y Piéron, M. (1996). Orientation des objectifs et participation à des activités sportives. Sport, 159/160, 82-89.

Maehr, M. L. y Nicholls, J. G. (1980). Culture and achievement motivation: a second look. In N. Warren (Ed.), Studies in cross-cultural psycbology, (pp. 221-267). New York: Academic Press.

Maher, M. L. y Braskamp, L. A. (1986). The motivation factor. A theory of personal investiment. Lexinton: Lexinton Books.

McGregor, H. A. y Elliot, A. J. (2002). Achievement goals as predictors of achievementrelevant processes prior to task engagement. Journal of Personality and Social Psychology, 94, 381-395.

Mitchell, S. (1996). Relationships between perceived learning environment and intrínsico motivation in middle school physical education. Journal of Teaching in Physical Education, 15, 369-383.

Muñiz, J. y Bartram, D. (2007). Improving international tests and testing. European Psychologist, 12, 206-219.

Muñiz, J. y Hambleton R. K. (2000). Adaptación de los tests de unas culturas a otras. Metodologia de las Ciencias del Comportamiento, 2, 129-149.

Newton, M., Duda, J. y Yin, Z. (2000). Examination of the psychometric properties of the Perceived Motivational Climate in Sport Questionnaire-2 in a sample of female athletes. Journal of Sports Sciences, 18, 275290.

Nicholls, J. (1984). Achievement Motivation: Conceptions of Ability, subjective experience, task choice, and performance. Psychological Review, 91, 328-346.

Nicholls, J. (1989). The competitive ethos and democratic education. Cambridge: Harvard University Press.

Ntoumanis, N. y Biddle, S. J. H. (1999). A review of motivational climate in physical activity. Journal of Sports Sciences, 17, 643- 665.

Nunnally, J. C. y Bernstein, I. J. (1995). Teoría psicométrica. Madrid: McGrawHill.

Osterlind, S. J. (1989). Constructing Test Items. Londres: Kluwer Academic Publishers.
Otero, F., González, J. A. y Calvo, A. (2012). Validation tools for measuring procedural and declarative knowledge and assessing decisions in school european football. Retos, 22, 65-69.

Papaioannou, A. (1994). Development of a questionnaire to measure achievement orientations in physical education. Research Quarterly for Exercise and Sport, 65, 11-20.

Papaioannou, A. y McDonald, A. I. (1993). Goal perspectives and purposes of physical education as perceived by Greek adolescents. Physical Education Review, 16, 41-48.

Papaioannou, A, Bebetsos, E Theodorakis, Y, Christodoulidis, T, y Kouli, O. (2006). Causal relationships of sport and exercise involvement with goal orientations, perceived competence and intrinsic motivation in physical education: A longitudinal study. Journal of Sports Sciences, 24, $367-382$

Papaioannou, A., Marsh, H. y Theodorakis, Y. (2004). A Multilevel Approach to Motivational Climate in Physical Education and Sport Settings: An Individual or a Group Level Construct? Journal of Sport y Exercise Psychology, 26(1), 90-119.

Papaioannou, A., Tsigilis, N., Kosmidou, E. y Milosis, D. (2007). Measuring perceived motivational climate in physical education. Journal of Teaching in Physical Education, 26, 236-259.

Pazo, C. I. y Tejada, J. (2012). The professional skills in Physical Education. Retos, 22, 5-8.

Peiró, C. y Sanchís, J. R. (2004). Las propiedades psicométricas de la versión inicial del cuestionario de orientación a la tarea y al ego (TEOSQ) adaptado a la educación física en su traducción al castellano. Revista de Psi cología del Deporte, 13, 25-39.

Ries, F. (2011). Physical self-concept in adolescents from Seville in relation with gender and the evolution of sport career. Retos, 19, 38-42.

Ruiz-Juan, F. y Piéron, M. (2013). Orientaciones de meta en Educación Física y nivel de actividad físico-deportiva en estudiantes mexicanos. Universitas Psychologica, 12(1).

Ruiz-Juan, F., Piéron, M. y Zamarripa, J. (2011). Versión española del “Task and Ego Orientation in Sport Questionnaire (TEOSQ)" adaptado a Educación Física. Estudios de Psicología, 32(2), 179-193.

Sánchez, P. M., Leo, F. M., Gómez, F. R., Sánchez, D., de la Cruz. E. y García, T. (2009). Orientations and motivational climates of the other significatives in young handball players from Extremadura. Retos, 16, 22-27.

Shumacker, R. E. y Lomax, R. G. (1996). A beginner's guide to structural equation modeling. Mahwah, NJ: Erlbaum.

Spaan, M. (2006). Test and item specifications development. Language Assessment Quarterly, 3, 71-79.

Van Der Horst, K., Paw, M., Twisk, J. y Van Mechelen, W. (2007). A Brief Review on Correlates of Physical Activity and Sedentariness in Youth. Medicine y Science in Sports y Exercise. 39, 1241-1250.

Walling, M. D. y Duda, J. L. (1995). Goals and their association with beliefs about success in and perceptions of the purpose of physical education. Journal of Teaching Physical Education, 14, 140-156.

Wang, C. K. J., Lim, B. S. C., Aplin, N. G., Chia, M., McNeill, M. y Tan, W. K. C. (2008). Students' Perceived Purposes of Physical Education in Singapore: Perspectives from a 2 × 2 Achievement Goals Framework. European Physical Education Review, 14, 51-70.

Wang, J., Biddle, S. y Elliot, A. (2007). The $2 \times 2$ achievement goal framework in a physical education context. Psychology of Sport and Exercise, 8(2), $147-168$.

Wang, J., Chia, W., Chatzisarantis, N. y Lim, C. (2010). Infuence of Perceived Motivational Climate on Achievement Goals in Physical Education: A Structural Equation Mixture Modeling Analysis. Journal of Sport y Exercise Psychology, 32, 324-338.

White, S. y Duda, J. (1994). The relationship of gender, level of sport involvement, and participation motivation to task and ego orientation. International Journal of Sport Psychology, 25, 4-18.

(Artículo recibido: 15-9-2012; revisado: 1-1-2013; aceptado: 10-1-2013) 
Anexo 1. Escala Achievement Goals Questionnaire (AGQ) en español

En una escala del 1 (Totalmente en desacuerdo) al 5 (Totalmente de acuerdo), dinos tu grado de desacuerdo o de acuerdo con las siguientes afirmaciones referentes a las clases de Educación Física.

En las clases de Educación Física:

1.Me siento muy bien cuando soy el único que puede hacer un ejercicio

2.Estoy completamente satisfecho cuando supero a los demás

3.Es importante para mí aprender un juego o habilidad para que los demás me quieran

4.Procuro evitar habilidades o juegos por los que se puedan burlar de mí

5.Mi meta constantemente es desarrollar mis habilidades

6.Siento éxito cuando soy el único que puede hacer una habilidad o juego

7.Frecuentemente me preocupa que me digan que no tengo ninguna habilidad

8.Estoy contento cuando desarrollo mis habilidades y los demás me valoran

9.Trato de participar y/o esforzarme más con el fin de aprender nuevas habilidades y juegos

10.Evito habilidades y juegos en los que no soy capaz de hacerlo bien

11.Me gusta aprender nuevas habilidades y juegos para ganarme la aprobación de los demás

12.Es importante para mí aprender nuevas cosas siempre

13.Me preocupa aparentar que no soy capaz de hacerlo bien

14.Cuando aprendo una habilidad quiero repetirla más veces

15.Trato siempre de intentar llevar la delantera a los demás en habilidades o juegos

16.Frecuentemente me preocupa que si intento hacer una actividad pueda parecer que no soy capaz de hacerlo bien

17.Quiero alcanzar un rendimiento alto para que los demás otros me quieran

18.Siempre intentaré superar a mis compañeros de clase

19.Me gusta hacer un ejercicio perfectamente para sentirme querido por los demás

20.Me gusta aprender nuevas habilidades sin importar la dificultad de las mismas

21.Suelo evitar ejercicios y juegos en los que puedo parecer incapaz

22.Me siento absolutamente exitoso cuando hago algo bien y los demás me den el visto bueno

23.Estoy satisfecho cuando los demás no pueden hacer algo tan bien como yo lo hago

24.Me gusta hacer un esfuerzo en ejercicios difíciles porque este es el camino para desarrollar mis habilidades 